\title{
EXTENSIÓN UNIVERSITARIA Y ESTRATEGIA DE OPERACIÓN
}

\author{
Univerrsity Extension anda Strategy of Operation
}

\author{
Fermin Francisco Chaiña Chura ${ }^{1}$
}

\begin{abstract}
RESUMEN
El propósito de esta investigación fue determinar las características del modelo de extensión universitaria; el problema: ¿Cuál es la naturaleza del modelo de extensión universitaria y de la estrategia de operación? La hipótesis: La socialización del conocimiento a la población se desarrolla en el marco del modelo de extensión universitaria centrado en la transferencia vertical. Se seleccionó una muestra intencional de diez universidades a nivel nacional; se analizó los documentos de gestión de estas instituciones, a través de la técnica del análisis de contenido y su guía. Los resultados indican que el $20 \%$ de universidades realizan la socialización del conocimiento a la población, conjugando el modelo de transferencia vertical y el modelo de extensión horizontal. El $80 \%$ de universidades realizan este proceso a través del modelo de transferenca vertical. La predominancia de misión y fines institucionales en un $80 \%$ y $60 \%$ respectivamente, significa que la universidad, sigue asumiendo funciones tradicionales. Solo el $20 \%$ de misiones y el $40 \%$ de los fines institucionales tienden a superar las funciones tradicionales conjugando con funciones comtemporáneas.
\end{abstract}

Palabras clave: Universidad, misión, fines, extensión universitaria, intervención.

\begin{abstract}
The purpose of this research was to determine the characteristics of the model of university extension; the problem: What is the nature of university extension model and operating strategy? A purposive sample of ten universities nationwide was selected; management documents of these institutions were analyzed through the technique of content analysis and guidance. The results indicate that $20 \%$ of universities conduct the socialization of knowledge towards the population, combining the vertical transfer model and the model of horizontal extension. Eighty ( $80 \%)$ of universities conduct this process through vertical transfer model. The predominance of mission and institutional purposes by $80 \%$ and $60 \%$ respectively; it means that the university continues assuming traditional roles. Only 20\% of missions and $40 \%$ of corporate goals tend to overcome traditional functions combined with contemporary functions.
\end{abstract}

Keywords: University, mission, goals, university extension intervention.

\section{INTRODUCCIÓN}

Se analiza las acciones de extensión que desarrolla la institución universitaria en el Perú, a fin de dinamizar esta función de educación no formal y contribuir al desarrollo humano sostenible. Hasta la actualidad, la extensión universitaria, se origina en la mente de autoridades, docentes, profesionales de la

\footnotetext{
${ }^{1}$ Doctor en Educación, Maestro en Administración de la Educación y Magister Scientiae en Desarrollo Rural. Docente de la Facultad de Ciencias Sociales de la Universidad Nacional del Altiplano. Puno-Perú.

Correoelectrónico:fchaiña1@hotmail.com
} 
universidad, no se identifica la demanda social de la población del ámbito de influencia de la universidad.

No se concibe la extensión universitaria como proceso holístico, que comprende las fases de: identificación de la demanda social de extensión, concepción de extensión, diseño de políticas de extensión, planificación de actividades, ejecución y evaluación. La extensión universitaria se realiza, sin coordinación con el sector institucional y sin participación de los interesados en el proceso de intervención. La interrogante fue: ¿Cuál es la característica del modelo de extensión universitaria y de la estrategia de operación? La hipótesis se planteó en los siguientes términos: La socialización del conocimiento a la población se desarrolla en el marco del modelo de extensión universitaria centrado en la transferencia vertical hacia la población del ámbito de influencia.

\section{MATERIALES Y MÉTODOS}

\section{Tipo y nivel de investigación}

El tipo de investigación es aplicado porque sus resultados contribuiran al desarrollo de las funciones universitarias, principalmente la de extensión; el nivel de investigación es descriptivo y analítico porque desarrolla el modelo de extensión.

\section{Población y muestra}

La población está compuesta por universidades nacionales y privadas del Perú, lo que constituyó el marco de muestreo; en la determinación del tamaño de muestra se utilizó el muestreo intensional de diez universidades en el marco de la ley $\mathrm{N}^{\circ} 23733$, entre el periodo de 2004 2014; se utilizó los planes estratégicos de estas universidades.

\section{Técnicas e instrumentos de colecta de información}

Para obtener la información necesaria a fin de desarrollar la hipótesis propuesta, se trabajó en base a segunda fuente, consistentes en documentos de gestión institucional; se utilizó la técnica del análisis de contenido y su instrumento la guía respectiva, con el que se recogió los datos necesarios; en forma complementaria se utilizó la observación directa.

\section{RESULTADOS Y DISCUSIÓN}

\section{Política de Extensión Universitaria}

La política institucional concentra la decisión de la autoridad expresada en lineamientos de política, lo que es aplicado por instancias operativas de la estructura organizacional y en sus relaciones con diferentes organizaciones de la sociedad, a fin de garantizar la misión institucional (Guillen, 1997; Jaramillo, 1997).

La universidad asume un conjunto de funciones, entre los que se destaca la formación profesional, la investigación y la extensión universitaria. La universidad en el Perú, estuvo regulado por la Ley Universitaria $\mathrm{N}^{\circ} 23733$, y últimamente, por la nueva Ley Universitaria $\mathrm{N}^{\circ} 30220$ a partir de 2014 , veamos algunos artículos de la primera norma referidos a la extensión universitaria:

En capítulo I: Disposiciones Generales, Artículo $1^{\circ}$ :

Las universidades están integrados por profesores, estudiantes y graduados. Se dedican al estudio, la investigación, la educación y de difusión del saber y la cultura, y a su extensión y proyección social.

En capítulo IX: De la Extensión y Proyección Universitaria. Artículo 68 ${ }^{\circ}$ :

Las universidades extienden su acción educativa a favor de quie- 
nes no son sus estudiantes regulares, en tal sentido organizan actividades de promoción y difusión de cultura general y estudios de carácter profesional, que pueden ser gratuitos o no, y que pueden conducir a una certificación.

Además, el Artículo $2^{\circ}$ referido a fines de la universidad, principalmente los incisos a y d; así como en el Artículo $3^{\circ}$ principios, en el inciso a, se refiere a la acción de extender sus servicios a la comunidad y promover su desarrollo integral.

En esta norma se dispone también lo siguiente: Establecen relaciones con las instituciones culturales, sociales y económicas con fines de cooperación, asistencia y conocimiento recíprocos. Participan en la actividad educativa y cultural de los medios de comunicación social y del Estado. Prestan servicios profesionales en beneficio de la sociedad y regulan estas acciones en su Estatuto de acuerdo con sus posibilidades y necesidades del país y la región que corresponden a su zona de influencia.

En consecuencia, la política de extensión universitaria que define el modelo de extensión, orienta la transferencia del conocimiento científico, tecnológico y humanístico a la población en el marco de una política de extensión, expresada en determinados principios y objetivos en un momento determinado. Lo referente a la nueva Ley Universitaria $N^{\circ} 30220$ se presenta en la discusión.

\section{Modelo de extensión universitaria}

En esta parte se analiza la tendencia del modelo de extensión universitaria en base a funciones específicas. La extensión universitaria es un medio para lograr la misión y los fines institucionales, vía socialización del conocimiento con el objeto de coadyuvar al bienestar de la población en general, así como el desarrollo del hombre en lo económico, social, cultural y político en lo específico (Vega Centeno, 2014).

Para el análisis del modelo de extensión, se construyó una tabla expresada en cifras absolutas y cifras relativas; con la siguiente regla de decisión: a) El proceso de socialización del conocimiento a la población - SCP es adecuada cuando se realiza a través de la "... comunicación horizontal y dialógica" (Freire, 1984); b) este proceso resulta regularmente adecuada, cuando se desarrolla a través de la conjugación de la transferencia vertical y la comunicación horizontal; y c) es inadecuada cuando la extensión se realiza a través de la transferencia vertical. La extensión universitaria, cuya función específica es orientar la SCP presenta las siguientes características:

Se observa, que el $20 \%$ de instituciones universitarias direcciona la SCP, conjugando el modelo de transferencia vertical y el modelo de extensión horizontal, por lo que se valora como regularmente adecuada en la escala de calificación; el $80 \%$ de universidades orientan este proceso de socialización a través del modelo de transferencia vertical, por lo que se valora como inadecuada; ninguna universidad presenta características del nivel adecuado en la SCP vía modelo de extensión horizontal, comunicativa y dialógica.

\section{Misión, fines institucionales y exten- sión universitaria}

Para el análisis de la misión, los fines institucionales y la extensión universitaria, se construyó una tabla con la siguiente regla: a) Son inadecuadas cuando direccionan hacia funciones tradicionales de universidad como enseñanza, investigación y extensión universitaria; b) son regularmente adecuadas cuando direccionan hacia funciones tradiciona- 
Tabla.1 Modelo de extensión universitaria y socialización del conocimiento a la población.

\begin{tabular}{lcc}
\hline \multicolumn{1}{c}{ Escala de Calificación } & $\mathbf{N}^{\circ}$ & $\%$ \\
\hline $\begin{array}{l}\text { Adecuada socialización del conocimiento a la población a través de la extensión } \\
\text { horizontal. }\end{array}$ & 00 & 00 \\
$\begin{array}{l}\text { Socialización del conocimiento a la población regularmente adecuada, vía } \\
\text { transferencia verticaly extensión horizontal. }\end{array}$ & 02 & 20 \\
$\begin{array}{l}\text { Inadecuada socialización del conocimiento a la población a través de la } \\
\text { transferencia vertical. }\end{array}$ & 08 & 80 \\
TOTAL: & $\mathbf{1 0}$ & $\mathbf{1 0 0} \%$ \\
\hline
\end{tabular}

Fuente: Elaborado en base a la muestra de diez universidades del Perú.

les conjugado con funciones contemporáneas y c) resultan adecuadas cuando direccionan funciones contemporáneas de universidad como la formación del potencial humano de valor estratégico que requiere la región y el país, la generación del conocimiento científico, tecnológico, humanístico de valor estratégico y la socialización del conocimiento a la población del ámbito de influencia, vía modelo de extensión horizontal y dialógica.

En la tabla 2, es evidente la predominancia de misión y fines institucionales del nivel inadecuado en un $80 \%$ y $60 \%$ respectivamente, significa que la universidad, sigue asumiendo funciones tradicionales como enseñanza, investigación y extensión universitaria; solo el $20 \%$ de misiones y el $40 \%$ de los fines institucionales tienden a superar las funciones tradicionales conjugando con funciones contemporáneas de universidad, por lo que se valora como regularmente adecuada. Entonces, resulta predominante el direccionamiento hacia funciones tradicionales en la universidad, expresados en la misión y fines institucionales.

Una función específica de la política universitaria de extensión es la socialización del conocimiento a la población, la misma que resulta inadecuada porque tiende a orientar este proceso a través de la transferencia vertical en un $90 \%$ de universidades; solo el $10 \%$ de instituciones universitarias orientan el proceso de divulgación del conocimiento en un nivel regularmente adecuado, debido a que la transferencia vertical es conjugado con una extensión horizontal, comunicativa y dialógica.

\section{DISCUSIÓN}

El estudio se discute en tres niveles: a) referente a la normatividad universitaria tanto de la ley $\mathrm{N}^{\circ} 23733$ derogada y la ley $\mathrm{N}^{\circ} 30220$, b) La institución universitaria y la extensión, y c) las estrategias de operación.

\section{Normatividad y extensión universitaria}

La Ley Universitaria $\mathrm{N}^{\circ} 23733$, derogada, cumplió funciones tradicionales de universidad y estas fueron: enseñanza, investigación y extensión, éste último a través del modelo de transferencia vertical. La nueva Ley Universitaria $N^{\circ} 30220$ en sus fines y funciones considera: "Proyectar a la comunidad sus acciones y servicios para promover su cambio y desarrollo; también considera, promover el desarrollo humano y sostenible en el ámbito local, regional, nacional y mundial; difundir el conocimiento universal en beneficio de la humanidad; servir a la comunidad y al desarrollo integral por un lado y por otro, dispone las funciones de extensión cultural y proyección social y contribuir al desarrollo humano". En las funciones docentes está vigente la proyección 
Fermin Francisco Chaiña Chura. Extensión Universitaria y Estrategia de Operación.

Tabla 2. Misión, Fines Institucionales y Extensión Universitaria

\begin{tabular}{lccccc}
\hline \multicolumn{1}{c}{$\begin{array}{c}\text { Componentes de la política } \\
\text { universitaria }\end{array}$} & $\begin{array}{c}\text { Regularmente Adecuado } \\
\mathbf{N}^{\circ}\end{array}$ & $\%$ & $\begin{array}{c}\text { Inadecuado } \\
\mathbf{N}^{\circ}\end{array}$ \\
\hline Misión & 02 & 20 & 08 & 80 \\
Fines & 04 & 40 & 06 & 60 \\
Extensión universitaria & 01 & 10 & 09 & 90 \\
\hline
\end{tabular}

Fuente: Elaborado en base a la muestra de diez universidades del Perú.

social (Ley N³0220. Art. 6, 7 y 79).

$\mathrm{La}$ responsabilidad universitaria

es el "...ejercicio de sus funciones: académicas, de investigación y de servicios de extensión y participación en el desarrollo nacional en sus diferentes niveles y dimensiones...". Así mismo, cuando la ley se refiere a los medios de promoción de la responsabilidad social universitaria concretiza en los estándares de acreditación, en las dimensiones, de investigación, de participación en el desarrollo social y servicios de extensión, ambiental e institucional respectivamente (Ley No30220: Art.124, 125).

En consecuencia, la Ley Universitaria $N^{\circ} 30220$ considera la extensión universitaria, en similares condiciones que la Ley derogada y aún peor en el marco de una concepción tradicional de universidad. No se refiere a la extensión universitaria en el marco de una concepción contemporánea de universidad dedicada a la socialización del conocimiento a la sociedad del ámbito de influencia, a la población que no tuvo la oportunidad de estudiar e informarse en el sistema de la universidad. Sí es prioritario en esta norma la calidad de formación profesional y la investigación en una universidad acreditada.

Para esta nueva ley universitaria, la extensión no tiene relevancia, porque de tener importancia, se hubiera creado una nueva estructura en el sistema de la universidad peruana, el Vicerrectorado de Extensión Universitaria, como se tiene por ejemplo en las universidades brasileras, entre otras.

\section{Universidad y la función de extensión}

La universidad como institución social pensante, como institución del saber, comprometida con el proceso de liberación de la sociedad, en términos de (Mejía, 2009) "... de la colonialidad del saber que implica una geopolítica del conocimiento", redefine permanentemente sus políticas, cumple funciones orientadas a la producción y socialización del conocimiento. Estas líneas de acción, requieren una política de ciencia y tecnología, una política de extensión, una política de formación del potencial humano que necesita la región y el país.

Esta perspectiva en términos operativos, requiere una concepción holística de la función de extensión universitaria, de una estrategia de operación como conjunto de componentes que se relacionan entre sí a través de acciones, desde la identificación de necesidades, aspiraciones, urgencias, que constituyen la demanda social para enfrentar los problemas existentes en las unidades de producción del ámbito de influencia de la universidad; a fin establecer soluciones para habilitar aptitudes en el uso de elementos técnicos y sociales que permitan mejorar el desempeño, desarrollando mejor sus actividades productivas, sociales y organizativas que le den vigencia en su núcleo local y dentro de la sociedad contemporánea (Ho, 2014).

\section{Estrategias de operación y operación de estrategias}

En lo referente a las estrategias de operación de la extensión universitaria en el proceso de intervención, es necesa- 
rio definir una concepción filosófica y un modelo de extensión, en este caso se trabaja dentro de un modelo horizontal y dialógica, donde el principal medio de comunicación es la dialogicidad, la deliberación participativa de la situación problemática; en el marco de una filosofía de extensión "ayudar a ayudarse" por un lado, y por otro lado, es necesario el diseño de estrategias de operación en el proyecto de extensión universitaria, como instrumento de gestión que guiará todo el proceso de extensión; en el momento siguiente de intervención, es necesario la operación de estrategias expresados específicamente en métodos de extensión universitaria, las mismas que pueden ser: individuales y/o grupales. Entre las primeras se tiene: las visitas de asesoramiento a predios familiares, la comunicación telefónica entre otros métodos individuales; entre los métodos de extensión grupales se tiene: La charla, el debate, el día de demostración de métodos, el día de demostración de resultados, el video, los grupos focales, entre otros componentes estratégicos, los mismos que permiten operar las estrategias de manera específica, en función al tipo de organización rural o urbano marginal con los que se trabaja.

\section{CONCLUSIONES}

Es evidente que, el 20\% de instituciones universitarias realizan la socialización del conocimientos a la población, conjugando el modelo de transferencia vertical y el modelo de extensión horizontal, por lo que se valora como regularmente adecuada; $\mathrm{el} 80 \%$ de universidades realizan este proceso de socialización a través del modelo de transferencia vertical, por lo que se valora como inadecuada; ninguna universidad presenta características del nivel adecuado en la socialización del conocimiento a la población vía modelo de extensión horizontal, comunicativa y dialógica.

La predominancia de misión y fines institucionales del nivel inadecuado en un $80 \%$ y $60 \%$ respectivamente, significa que la universidad, sigue asumiendo funciones tradicionales; solo el $20 \%$ de misiones y el $40 \%$ de los fines institucionales tienden a superar las funciones tradicionales conjugando con funciones contemporáneas de universidad, por lo que se valora como regularmente adecuada.

La extensión universitaria cuya función fundamental es la socialización del conocimiento a la población resulta inadecuada, porque tiende a orientar este proceso a través de la transferencia vertical en un $90 \%$ de universidades; solo el $10 \%$ orienta el proceso de divulgación del conocimiento a la población en un nivel regularmente adecuado, debido a que la transferencia vertical es conjugado con una extensión horizontal, comunicativa y dialógica.

\section{REFERENCIAS BIBLIOGRÁFICAS}

Freire, P. (1984). ¿Extensión o comunicación? la concientización en el medio rural. 13a. Edición. D.F. México: Siglo XXI.

Ho, R. (2014). Extensión rural y asistencia técnica en el sector campesino: experiencias del periodo 1997 - 2013 lecciones y propuestas. Lima, Perú: Kinkos.

Guillén, J. (1997). Pautas y propuestas de política Universitaria. Documento aprobado en Consejo Universitario del 11 de febrero de 1997. Arequipa, Perú: UNSA.

Jaramillo, C. (1997). La gerencia del desarrollo social: un reto para el siglo XXI. México: Comercio Exterior.

Mejía, J. (2009). Sociedad y conocimiento los desafíos de la sociología contemporá- 
nea. Lima, Perú: Fondo Editorial FCSUNMSM.

Vega Centeno, M. (2014). Del desarrollo esquivo al desarrollo sostenible: ensayos sobre la innovación, el desarrollo, el crecimiento y la sostenibilidad. Lima, Perú: Fondo editorial PUCP.

Texto Ley Universitaria N ${ }^{\circ} 23733$ y Texto Ley Universitaria $\mathrm{N}^{\circ} 30220$. 